\title{
Electrical dependencies of optical modulation capabilities in digitally addressed parallel aligned liquid crystal on silicon devices
}

\author{
Francisco Javier Martínez, ${ }^{a, b}$ Andrés Márquez, ${ }^{a, b, *}$ Sergi Gallego, ${ }^{a, b}$ Manuel Ortuño, ${ }^{a, b}$ Jorge Francés, ${ }^{a, b}$ \\ Augusto Beléndez, ${ }^{a, b}$ and Inmaculada Pascual ${ }^{b, c}$ \\ aUniversidad de Alicante, Dept. de Física, Ing. de Sistemas y T. Señal, Ap. 99, Alicante E-03080, Spain \\ buniversidad de Alicante, I.U. Física Aplicada a las Ciencias y las Tecnologías, Ap. 99, Alicante E-03080, Spain \\ 'Universidad de Alicante, Dept. de Óptica, Farmacología y Anatomía, Ap. 99, Alicante E-03080, Spain
}

\begin{abstract}
Parallel aligned liquid crystal on silicon (PA-LCoS) displays have found wide acceptance in applications requiring phase-only modulation. Among LCoS devices, and PA-LCoS as a specific case, digital addressing has become a very common technology. In principle, modern digital technology provides some benefits with respect to analog addressing such as reduced interpixel cross-talk, lower power consumption and supply voltage, gray level scale repeatability, high programmability, and noise robustness. However, there are also some degradating issues, such as flicker, which may be enhanced. We analyze the characteristics of the digital pulse width modulated voltage signals in relation to their effect on the optical modulation capabilities of LCoS displays. We apply calibration techniques developed in our laboratory, basically the classical linear polarimeter extended to take into account the existence of flicker. Various digital sequence formats are discussed, focusing the analysis on the variations in the magnitude of the applied voltages across the LC layer. From this analysis, we obtain how to amplify the retardance dynamic range and how to enhance linearity in the device without enhancing flicker and without diminishing the number of available quantization levels. Electrical configurations intended for phase-only and intensity modulation regimes, useful in diffractive optics, are given. ๑ 2014 Society of PhotoOptical Instrumentation Engineers (SPIE) [DOI: 10.1117/1. OE.53.6.067104]
\end{abstract}

Keywords: liquid crystal on silicon displays; parallel aligned; retardance measurement; phase-only modulation; spatial light modulation; flicker; diffractive optics; pulse width modulation.

Paper 140570P received Apr. 4, 2014; revised manuscript received May 20, 2014; accepted for publication May 22, 2014; published online Jun. 24, 2014.

\section{Introduction}

Liquid crystal (LC) microdisplays have found widespread use as spatial light modulators (SLM) in applications such as in diffractive optics, ${ }^{1}$ optical storage, ${ }^{2}$ optical metrology, ${ }^{3}$ reconfigurable interconnects, ${ }^{4,5}$ or quantum optical computing. ${ }^{6}$ Among microdisplays, liquid crystal on silicon (LCoS) devices have become the most attractive system for these applications due to their very high spatial resolution and very high light efficiency. ${ }^{7-9}$ LCoS displays are technologically complex devices where the electrical signal applied controls the optical transmission in each pixel. A proper characterization and configuration of the main electrical parameters are crucial to generate the required optical modulation regimes in SLM applications, as exemplified by $\mathrm{Lu}$ and Saleh ${ }^{10}$ with the previous analog liquid crystal displays (LCDs) taking into account the applied voltage dynamic range and bias voltage. Interplay between electrical and optical performance parameters has also been useful for identifying the origin for degradation effects in LCDs, such as the anamorphic and frequency dependent modulation, ${ }^{11}$ also reported in both digital ${ }^{12}$ and analog ${ }^{13} \operatorname{LCoS}$ devices. An additional degradation effect detected in LCoS displays by several authors ${ }^{14-17}$ is that they produce phase flicker and/ or depolarization, especially the ones with a digital backplane $^{18}$ due to the pulsed nature of the voltage signal

*Address all correspondence to: Andrés Márquez, E-mail: andres.marquez@ ua.es addressed. ${ }^{8,9,19,20}$ Among the different LC layer geometries, parallel aligned LCoS (PA-LCoS) devices are especially interesting since they allow easy operation as phase-only devices without coupled amplitude modulation. ${ }^{8,9}$ No depolarization operation is also available; however, they may still exhibit phase fluctuations. ${ }^{18}$ Recently, we proposed a method to characterize not only the linear retardance but also the amount of phase flicker in the optical signal ${ }^{21}$ valid for parallel aligned LC devices. This method, the extended linear polarimeter, has demonstrated its applicability to PA-LCoS displays $^{21}$ exhibiting phase flicker; therefore, it can be used in the analysis of the influence of the electrical parameters on the optical modulation performance properties of LCoS displays. This method is an elaboration of the classical linear polarimeter which may be found to measure the birefringence in $\mathrm{LCs}^{22}$ and in waveplates. ${ }^{23}$

As described by Lueder, ${ }^{24}$ pixel circuitry in the LCoS backplane ${ }^{8,24-26}$ can be based on dynamic random-access memory (DRAM) or on static random-access memory (SRAM), which is accordingly related to the differentiation between analog and digital addressing. ${ }^{27,28}$ If a memory is provided at the pixel in the form of an analog storage capacitor (DRAM) or digital memory circuit (SRAM), the array can be frameaddressed, i.e., all pixels are addressed simultaneously. This has the advantage of reducing the low frequency flicker of the line-addressed arrays, which was typical in LCDs. ${ }^{11}$ 
Various reasons have motivated the introduction of digital backplanes in LCoS technology. The digital scheme is usually more stable than analog and shows a repeatable performance. Field inversion is possible at the kilohertz range (e.g., for each modulation pulse) without image retention. The scheme does not suffer from electrical cross-talk (i.e., no low pass filtering of the signal). ${ }^{29}$ A lower power consumption and a lower supply voltage are possible with the digital backplane. ${ }^{8,20}$ Digital driving can control the precise gray level scale. Another advantage of pulse modulation is that the LC is driven between zero and saturation voltages in a time multiplexed fashion, ${ }^{26-28}$ so that the slow response of switching between adjacent voltage states is avoided. The nematic LC responds to the root-mean-square (RMS) of the applied waveform integrated over the switching time of the LC. However, the time varying AC modulation of the driving voltage is partially transferred to the LC and produces a temporal fluctuation or flicker of the reflected light as described in Refs. 18-20, i.e., the electro-optical response of the SLM is not constant over the frame period. If the digital sequence format is properly chosen, fluctuations can be significantly decreased to acceptable levels. ${ }^{21}$ To this goal, a proper characterization not only of the phase modulation but also of the amount of phase flicker in the optical signal needs to be done. This reasoning is not restricted to PA-LCoS devices, but it can be applicable in general to electro-optic variable linear retarders such as the novel device proposed by Ramirez et al. ${ }^{30}$ Apart from the sequence format, other options (applied binary voltage value, selection of pulse codes, and number of gray scale levels) offered by digital addressing need to be analyzed to produce proper phase depth, enhanced linearity, number of quantization levels available, etc.

In this work, we take advantage of the ease of implementation and the effectiveness of the calibration method proposed in Ref. 21 to characterize the basic electrical parameters controlling the digital signal addressed to a PALCoS display and their effect on the retardance dynamic range and the magnitude of the flicker. Preliminary results were presented in Ref. 31. This enables us to produce electrical configurations specifically optimized for phase-only or for amplitude-mostly modulation regimes.

\section{Characterization Using the Extended Linear Polarimeter}

In the following, we introduce the extended linear polarimeter proposed in Ref. 21, where the linear retarder under test, in our case the PA-LCoS, is situated between two linear polarizers illuminated with a monochromatic laser source. In the analysis shown in Ref. 21, it was demonstrated that the classical technique based on measuring the intensity with a radiometer at the exit of the system for the parallel and crossed configurations of the polarizers, both oriented at 45 or $-45 \mathrm{deg}$ with respect to the neutral lines of the waveplates, is not only a simple method but also the most robust to misalignments and other types of noise sources in the system. With respect to the classical technique, the extended linear polarimeter ${ }^{21}$ enables us to characterize the retardance magnitude in the presence of retardance instabilities or flicker. Other techniques may also be applied; ${ }^{32,33}$ however, the present method is very straightforward to implement and accurate enough for the study to be done in this work.

In the case of instabilities or fluctuations in the linear retardance, we demonstrated ${ }^{21}$ that as a first approximation for PA-LCoS displays we may consider a model with a linear variation of retardance with time $\Gamma(t)$. In this case, the averaged parallel and crossed polarizers intensities become

$\left\langle I_{\mathrm{OUT}}^{\|}\right\rangle=\frac{I_{0}}{2}\left[\frac{\sin a}{a} \cos \bar{\Gamma}\right]$,

$\left\langle I_{\text {OUT }}^{\perp}\right\rangle=\frac{I_{0}}{2}\left[\frac{\sin a}{a} \cos \bar{\Gamma}\right]$,

where $\bar{\Gamma}$ and $a$ are the values for the average retardance and its flicker fluctuation amplitude, respectively. When combining the two expressions, we obtain

$\frac{\left\langle I_{\text {OUT }}^{\|}\right\rangle-\left\langle I_{\text {OUT }}^{\perp}\right\rangle}{\left\langle I_{\text {OUT }}^{\|}\right\rangle+\left\langle I_{\text {OUT }}^{\perp}\right\rangle}=\frac{\sin a}{a} \cos \bar{\Gamma}$,

where we see that the cosine term becomes modulated by the presence of a sinc function dependent on the magnitude of the fluctuation $a$. This sinc function represents the deviation from the classical expression typically used to obtain the linear retardance value. ${ }^{21}$ Eventually, the average retardance $\bar{\Gamma}$ is obtained by inverting Eq. (3)

$\bar{\Gamma}=\cos ^{-1}\left(\frac{\left\langle I_{\mathrm{OUT}}^{\|}\right\rangle-\left\langle I_{\mathrm{OUT}}^{\perp}\right\rangle /\left\langle I_{\mathrm{OUT}}^{\|}\right\rangle+\left\langle I_{\mathrm{OUT}}^{\perp}\right\rangle}{\sin a / a}\right)$.

In the case when no fluctuations exist ( $a=0 \mathrm{deg}$ ), then Eqs. (1)-(4) simplify into the classical results. We note that Eq. (4) returns the wrapped phase values in the range 0 to $180 \mathrm{deg}$, which is the definition domain for the $\cos ^{-1}$ function. In general, the average retardance $\bar{\Gamma}$ and the fluctuation amplitude $a$ are coupled in Eqs. (1)-(4), yet at the maxima and minima (the extremals) the retardance value is known; it is a multiple of $180 \mathrm{deg}$ ( 0 or $180 \mathrm{deg}$ in the wrapped domain). At these points, we may calculate the value for the fluctuation amplitude $a$ and then it can be introduced in Eq. (4) to produce a more accurate estimation of the average retardance $\bar{\Gamma}$ out of the extremals (what in Fig. 4 will be called the "corrected" values). To obtain $a$, it was demonstrated that at the extremals, the absolute retardance difference $\Gamma_{\text {diff }}$ between the calculated and the true retardance values is

$\Gamma_{\text {diff }}=\cos ^{-1}\left(\frac{\sin a}{a}\right)$.

By inverting the set of numerical values $\left(\Gamma_{\text {diff }}, a\right)$ provided by Eq. (5), we produce a fitting function enabling us to calculate $a$ as a function of the value measured for $\Gamma_{\text {diff. }}$ This fitting function was calculated in Ref. 21 for a fluctuation amplitude $a$ interval covering the range ( $0 \mathrm{deg}, 110 \mathrm{deg}$ ) and is given by the linear expression 


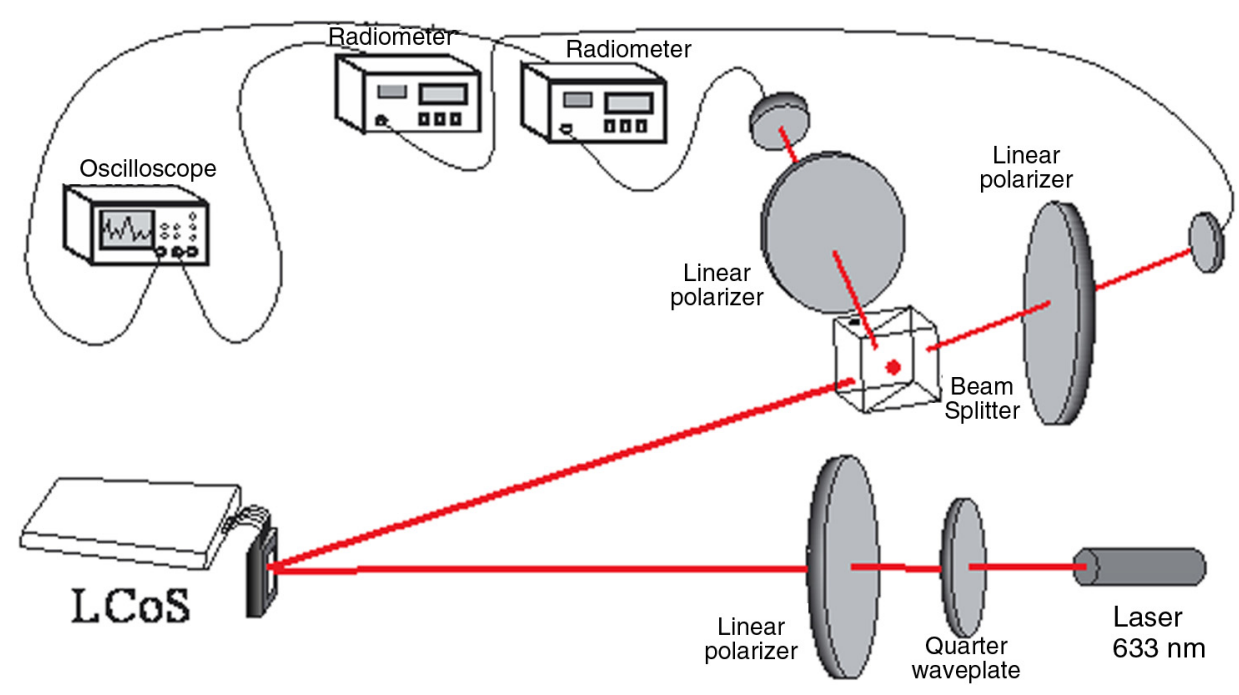

Fig. 1 Experimental setup used to measure the linear retardance as a function of the applied voltage (gray level) for a parallel aligned liquid crystal on silicon (LCoS). The setup allows both to measure average and instantaneous values.

$a=1.7795 \Gamma_{\text {diff }}$

In Fig. 1, we present the experimental setup that we use in this work to obtain the parallel and crossed polarizers intensities. The unexpanded beam from a He-Ne laser (633-nm wavelength) is incident onto a linear polarimeter where the reflective $\mathrm{LCoS}$ is the linear retarder to be measured. A quarter wavelength waveplate is added after the laser so that enough light passes through the first polarizer irrespective of the orientation of its transmission axis. We consider quasiperpendicular incidence at a 3-deg incidence angle onto the LCoS. We introduce one nonpolarizing cube beam splitter (NPBS) (model 10BC16NP.4, from Newport) to enable amplitude division with a 50/50 ratio of the reflected beam so that crossed and parallel intensities can be measured simultaneously (thus, two radiometers are introduced). This further enables synchronized measurement of the instantaneous parallel and crossed polarizers intensity values simply by connecting the two radiometers to the two channels of an oscilloscope (shown in this figure). The same setup is, therefore, valid for the characterization of the average and the instantaneous values. We note that in Ref. 21, we showed that insertion of NPBS did not introduce significant polarization effects in the calibration when perpendicular incidence onto the beam splitter is considered as will be the case in this paper.

\section{Digital Electrical Signal Addressing Parameters}

Let us provide the basic driving procedure to understand the general parameters configuring the pulsed signal addressed in digital backplane LCoS displays. There are two steps: $:^{23,25}$ data addressing to the pixel SRAM memory in the backplane and LC driving. First, the input signal is processed by the controller and turned into bit-plane format. In this step, a look-up table (LUT) is used to determine the correct bit sequence for a target gray level. The first bit plane image is written to the pixel SRAM of the digital backplane, where the pixel memory has only "on" or "off" information. There may be a master and a slave memory enabling data to be transferred to a pixel at the same time that the previously loaded data are being displayed. After the data-addressing step is finished, the next step is to drive the LC where three voltage signal values, $V_{0}$ and $V_{1}$, low and high voltage signals on the pixel backplane, and $V_{\text {ITO }}$, on the ITO transparent electrode on the glass substrate, are used. These voltages are applied to all pixels of the display area at the same time so that every pixel is driven by the same condition. The $V_{0}$ and $V_{1}$ signals are selected by the SRAM information of the pixel. Then, the driving voltage across the LC layer $V_{\mathrm{LC}}$ is given by $V_{\text {ITO }}-V_{1}$ or $V_{\text {ITO }}-V_{0}$, in the following $V_{\text {bright }}$ and $V_{\text {dark }}$, respectively. A schematic diagram for the absolute value of the digital signal $V_{\mathrm{LC}}$ is shown in Fig. 2 (upper line), where the amplitudes for $V_{\text {bright }}$ and $V_{\text {dark }}$ are represented. In this driving scheme, the LC layer is driven by high frequency that approximates a square wave running at several kilohertz. The nematic LC responds to the RMS value of the driving voltage over the switching time of the LC and the residual $\mathrm{AC}$ modulation is responsible for the flicker described in digital LCoS devices, represented in Fig. 2 (lower line). This is why it is generally understood that flicker noise can be minimized by high modulation rates. ${ }^{25,34}$

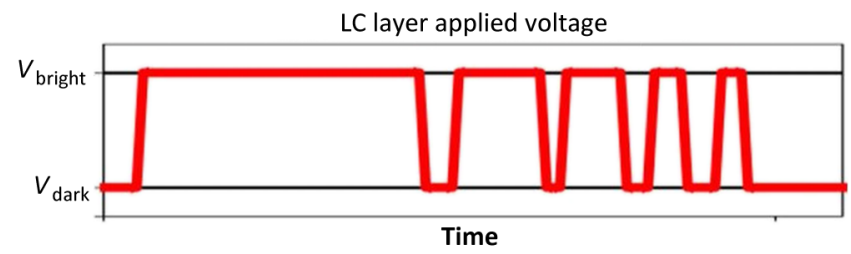

Liquid crystal response

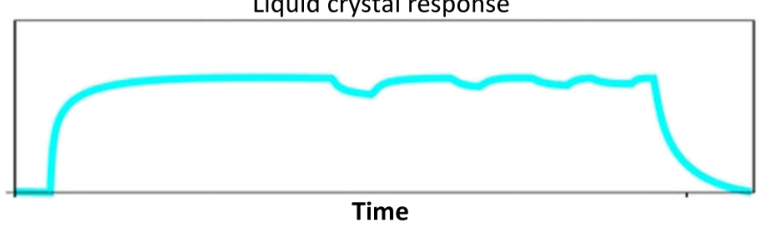

Fig. 2 Schematic diagram for the different voltage magnitudes involved in the digital addressing of the LCoS. Upper line driving voltage $V_{\mathrm{LC}}$ applied across the LC layer. Lower line voltage as seen by the LC molecules. 


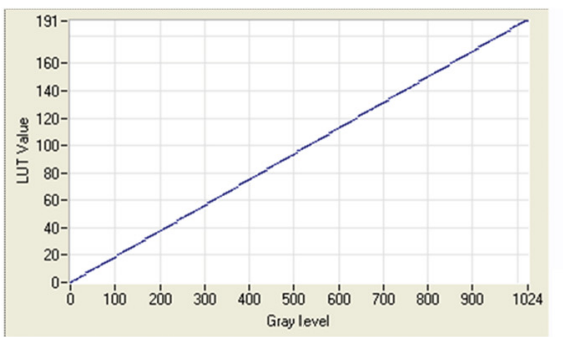

(a)

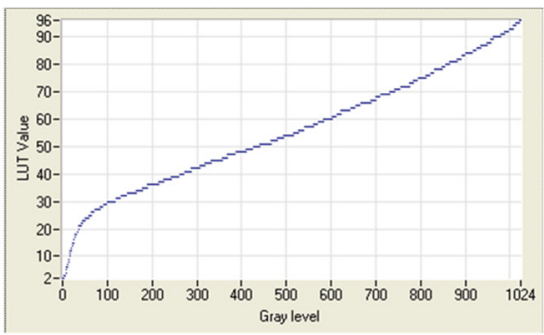

(b)

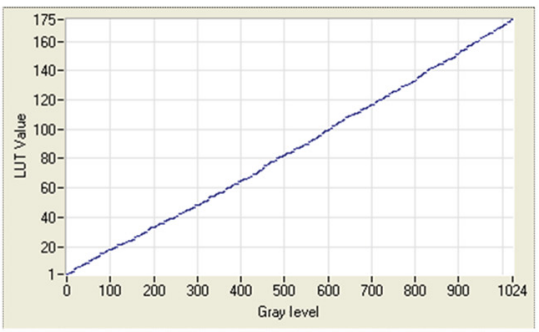

(c)

Fig. 3 Gamma curves for the PLUTO LCoS for the configuration files: (a) "5-5 default ramp," (b) "5-5 linear 2pi 633 nm," and (c) "5-5 linear 2pi 405 nm."

The analysis in this paper deals with a digitally addressed phase-only LCoS, specifically the electrically controlled birefringence LCoS display distributed by the company HOLOEYE (Berlin, Germany). It is an active matrix reflective mode device with $1920 \times 1080$ pixels and a 0.7 in. diagonal named the PLUTO SLM, with a pixel pitch of $8.0 \mu \mathrm{m}$ and a fill factor of $87 \%$. The signal is addressed via a standard digital visual interface signal. By means of the RS-232 interface and its corresponding provided software, we have access to the basic electrical parameters of the device ${ }^{19,20}$ previously described, such as the digital addressing sequence format (bit planes), the gamma curve, and the voltage dynamic range of the pulse width modulated (PWM) signal (through two digital potentiometers), i.e., voltages $V_{\text {bright }}$ and $V_{\text {dark }}$.

The software for the SLM is provided with a series of configuration files corresponding to two different digital addressing sequences: the so-called 18-6 and 5-5 digital sequences where the first number indicates the quantity of "equally weighted" bit planes and the second number indicates the quantity of "binary" bit planes. ${ }^{19}$ This translates into a number of distinct voltage levels as follows: $(18+1) \times$ $2^{6}=1216$ for the sequence " $18-6$ " and $(5+1) \times 2^{5}=192$ for the sequence " $5-5$." The larger number of bit planes makes the sequence 18-6 longer than the one corresponding for the sequence 5-5. In principle, the shorter the sequence, the smaller the flicker, ${ }^{19}$ but on the other side, a larger sequence provides a larger number of quantization levels. For each of the sequences, we find a series of configuration files which have been, in principle, optimized by the vendor for different wavelengths to enable SLM users a fast access to a proper operating configuration for the device adapted to the specific application and illuminating wavelength. In general, these configuration files enable phase-only operation with a $2 \pi$ linear phase dynamic range for the specified wavelength. To this goal, the digital potentiometer values and the gamma curve, which corresponds to the LUT, need to be properly selected.

In Sec. 2, we have presented an efficient and effective technique able to discriminate, in terms of the average retardance dynamic range and the magnitude of the flicker, between the digital addressing sequences given with the software and driver electronics. Finer adjustment of the retardance dynamic range and its linearity is then feasible by modifying the gamma curve and changing the digital potentiometers values. The simplicity of the linear polarimeter setup on which the method is based allows SLM users to have access to adapt the calibration to their specific working geometry and illuminating wavelength, adding novel possibilities to the configuration files provided by the vendor.

In the rest of this paper, we focus on sequence 5-5 since it produces less flicker than sequence 18-6, as previously commented on and as already demonstrated in a number of papers. ${ }^{19,35}$ By applying different gamma curves and different $V_{\text {bright }}$ and $V_{\text {dark }}$ voltages to a specific sequence we may obtain very different retardance versus gray level curves. In Figs. 3(a)-3(c), we show three examples of gamma curves for the 5-5 sequence, corresponding, respectively, to the configuration files named "5-5 default ramp," "5-5 linear 2pi $633 \mathrm{~nm}$," and "5-5 linear 2pi 405 nm." As we will show in Sec. 4.1, the first configuration provides the largest voltage modulation range available, thus enabling us to estimate the maximum retardance dynamic range provided by the LCoS unit. The second and third configurations span a retardance range of $2 \pi$ and $\pi$ radians, respectively, which are the necessary ranges to produce, respectively, a phase-only regime and an amplitude-mostly regime when using a variable linear retarder.

Figure 3(a) shows a linear mapping between the input and output values where all the 192 possible values associated with the 5-5 sequence are available. In Fig. 3(b), the mapping is highly nonlinear, especially for lower gray levels, and only 95 out of the 192 possible values are available. In Fig. 3(c), the mapping is rather linear and a large quantity of gray levels, 175 , are available.

With respect to the voltages $V_{\text {bright }}$ and $V_{\text {dark }}$, we note that both the configuration file "5-5 default ramp" and "5-5 linear 2pi $633 \mathrm{~nm}$ " have the same values, which are $V_{\text {bright }}=$ $3.82 \mathrm{~V}$ and $V_{\text {dark }}=0.03 \mathrm{~V}$. In the case of the configuration "5-5 linear 2pi $405 \mathrm{~nm}$," these values are $V_{\text {bright }}=2.02 \mathrm{~V}$ and $V_{\text {dark }}=1.36 \mathrm{~V}$. A change in these values affects the voltage amplitude of the PWM pulses, thus affecting the accumulated voltage experienced by the LC molecules in a whole frame period. We note that the largest voltage range possible is 3.82 to $0.03 \mathrm{~V}$.

\section{Results and Discussion}

\subsection{Initial Configurations}

Some additional details are necessary to complement the experiment description given in relation to Fig. 1. The intensities for both the parallel and crossed polarizer measurements have been obtained with the input polarizer transmission axis at $+45 \mathrm{deg}$ to the vertical of the laboratory, which is the $X$-axis of our reference system. We note that the director for the LC (i.e., the extraordinary axis) in the LCoS is oriented along the horizontal, i.e., at +90 deg with respect 


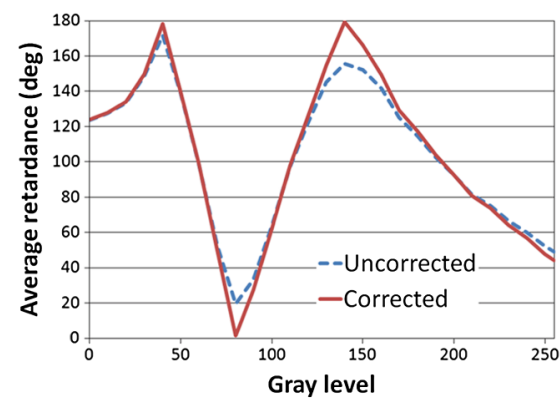

(a)

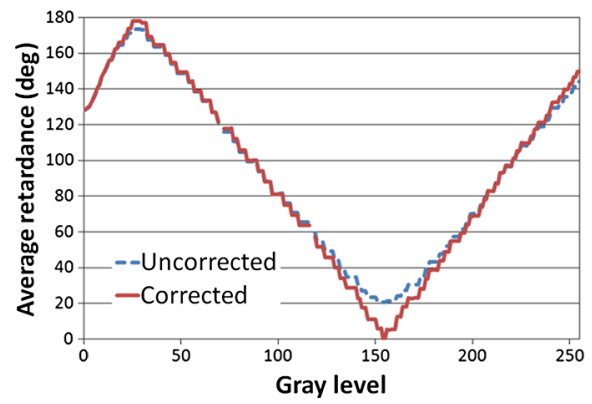

(b)

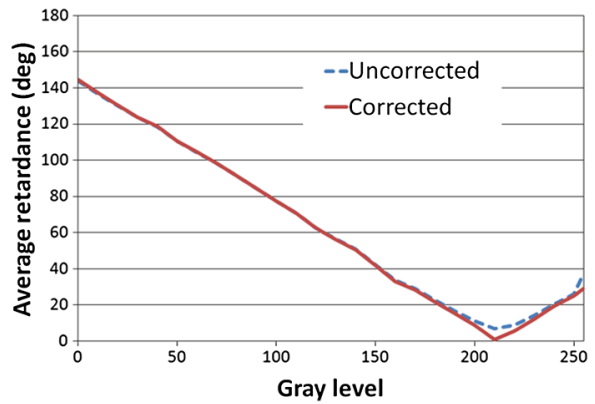

(c)

Fig. 4 Retardance (wrapped values) calculated using the classical approach (uncorrected curves) and the extended calibration method (corrected curves) for the configurations: (a) "5-5 default ramp," (b) " $5-5$ linear 2pi $633 \mathrm{~nm}$," and (c) "5-5 linear 2pi $405 \mathrm{~nm} . "$

to the $X$-axis. The director axis in nematic filled devices, as it is the case for our LCoS, typically corresponds to the slow axis. Typically, the angular uncertainty on the orientation of the input and output polarizers with respect to the neutral lines of the LCoS can be considered to be $\pm 1 \mathrm{deg}$.

In Fig. 4, we plot the retardance (wrapped values) calculated using the classical approach (uncorrected curves), which considers no flicker, and the extended calibration method (corrected curves). The plots in Figs. 4(a)-4(c) correspond, respectively, to the configurations "5-5 default ramp," "5-5 linear 2pi 633 nm," and "5-5 linear 2pi 405 nm." From the retardance difference at the extremals in the uncorrected curves, and applying the polynomial in Eq. (6), the fluctuation amplitude $a$ can be calculated and the corrected curves are obtained. These values are presented in Table 1. We see that the fluctuation amplitude increases with the applied gray level: for example, for the configuration " $5-5$ default ramp," the successive extremals exhibit fluctuation amplitudes of 14, 34, and $43 \mathrm{deg}$.

If the retardance is unwrapped in Fig. 4(a), we obtain a dynamic range of about 560 deg, exceeding the necessary $360 \mathrm{deg}$ for phase-only modulation. We observe from Fig. 4(a) that the uncorrected and corrected retardance curves only deviate at values which are multiples of $180 \mathrm{deg}$. We also see that the retardance presents a nonlinear relation with the gray level. In Fig. 4(b) for the configuration "5-5 linear 2pi $633 \mathrm{~nm}$," the retardance dynamic range is slightly higher than $360 \mathrm{deg}$, and the relation with the gray level is very

Table 1 Fluctuation amplitude a (third column) calculated for three electrical configuration files (first column), using the values for the retardance difference (second column) at the extremals for the parallel (or crossed) polarizers intensity curves as given by the calibration method in Ref. 21.

\begin{tabular}{lcc}
\hline Configuration & $\Gamma_{\text {diff }}($ deg $)$ & $a$ (deg) \\
\hline 5-5 default ramp & $7.9(\mathrm{GL} \mathrm{40)}$ & 14 \\
& $19.2(\mathrm{GL} \mathrm{80)}$ & 34 \\
& $24.5(\mathrm{GL} \mathrm{140)}$ & 43 \\
$5-5$ linear 2pi 633 nm & $6.6(\mathrm{GL} \mathrm{26)}$ & 12 \\
$5-5$ linear 2pi 405 nm & $20.6(\mathrm{GL} \mathrm{155)}$ & 36 \\
\hline
\end{tabular}

linear. Therefore, the gamma curve in Fig. 3 and the $V_{\text {bright }}$ and $V_{\text {dark }}$ values have been properly tuned and this configuration may be used for phase-only operation: this requires incident light linearly polarized along the molecular director of the LCoS (along the horizontal). In Fig. 4(c), the curve shows a retardance range o about $180 \mathrm{deg}$. This range is appropriate to obtain amplitude-mostly operation as we showed in Fig. 6(b) in Ref. 31.

\subsection{Control of the Modulation Range and Flicker}

In Sec. 3, we described the various parameters governing the magnitude and structure of the digital signal addressed. In this section, we present the effect observed when the values in the parameters are changed and how this can be used to control the modulation range to our benefit. We take advantage of the fact that the software interface for the digital LCoS model we use enables us to change the values in these parameters, in particular $V_{\text {bright }}$ and $V_{\text {dark }}$. We will consider the gamma curve and sequence corresponding to configuration " $5-5$ default ramp" which provides the larger dynamic modulation range, as shown in Fig. 4(a).

We want to study the effect of the magnitude of $V_{\text {bright }}$ and $V_{\text {dark }}$ on the retardance value. To start with we want to remove the pulsed nature of the digital signal addressed. To this goal, $V_{\text {bright }}$ and $V_{\text {dark }}$ must be equal. In fact, the minimum difference available with our LCoS device turns out to be $0.06 \mathrm{~V}$, i.e., slightly different from zero.

In Fig. 5, we show the instantaneous retardance values, measured with the setup in Fig. 1, for a series of configurations with $V_{\text {bright }} \cong V_{\text {dark }}$ and at gray level 100 . The first and

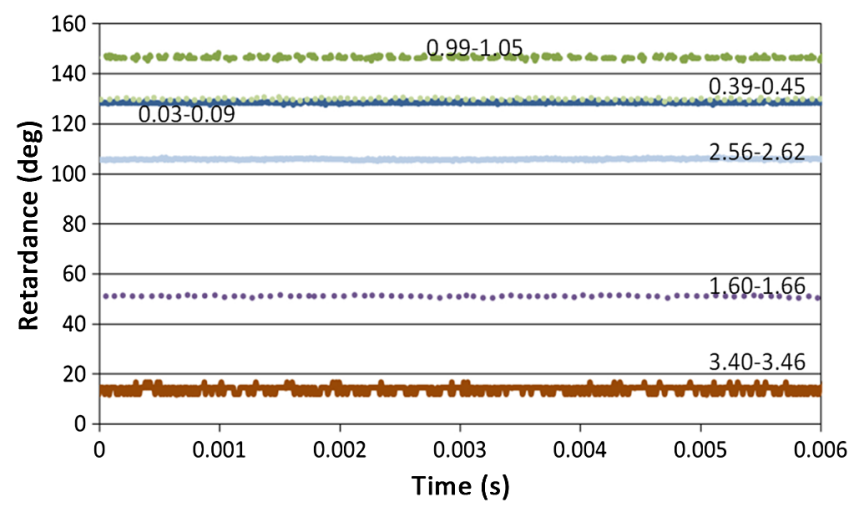

Fig. 5 Retardance temporal evolution for gray level $=100$ for $V_{\text {dark }} \cong V_{\text {bright }}$. 
second values in each pair in the legend correspond, respectively, to $V_{\text {dark }}$ and $V_{\text {bright }}$, ranging from $(0.03 \mathrm{~V}, 0.09 \mathrm{~V})$ to $(3.40 \mathrm{~V}, 3.46 \mathrm{~V})$, thus covering a wide voltage range. We see that the curves show no flicker, in agreement with the fact that the pulse character in the digital signal is lost: both the on and off bits addressed have equal voltage amplitudes. The fact that there are no temporal instabilities is also indicative that other origins for the flicker, such as thermal noise, can be disregarded. When applying other gray levels, the retardance values obtained remain the same and stable with time. We note that there is a threshold value below which the molecules do not react. This can be seen because of the overlap between the retardance curves for 0.03 and $0.39 \mathrm{~V}$. For higher voltage values, retardance curves start to evolve and do not overlap.

Next, let us consider different values for the parameters $V_{\text {dark }}$ and $V_{\text {bright }}$, so that retardance evolves as a function of gray level. The goal is to analyze how the retardance dynamic range as a function of gray level is affected. It is important to keep in mind that, according to the explanation in Sec. 3, the time structure for the pulsed sequence associated with each gray level is not affected by the changes in $V_{\text {dark }}$ and $V_{\text {bright }}$ and only the accumulated applied voltage must vary.

In Figs. 6(a) and 6(b), we plot the average retardance as a function of gray level when $V_{\text {dark }}$ and $V_{\text {bright }}$ are, respectively, changed. We note that in Figs. 6(a) and 6(b), voltages $V_{\text {bright }}$ and $V_{\text {dark }}$ are fixed, respectively, at 3.82 and $0.03 \mathrm{~V}$, which are the values for the results shown in Fig. 4(a). In Fig. 6(a), as we increase $V_{\text {dark }}$ the resulting effect is the increase of the accumulated voltage applied associated with each gray level: maxima in the curves shift to lower gray levels as we increase $V_{\text {dark }}$. At high applied voltages (high gray levels), we observe a saturation process and all the curves tend to a common average retardance value of about $20 \mathrm{deg}$. At gray level zero, the increase of $V_{\text {dark }}$ changes the starting retardance value which, combined with the saturation process, results in a reduction of the total retardance range. We note that the retardance at gray level zero is equal to the value obtained in the previous Fig. 5 for equal $V_{\text {dark }}$ voltages, thus indicating that the pulsed sequence associated with gray level zero has all the bits in the off-state.

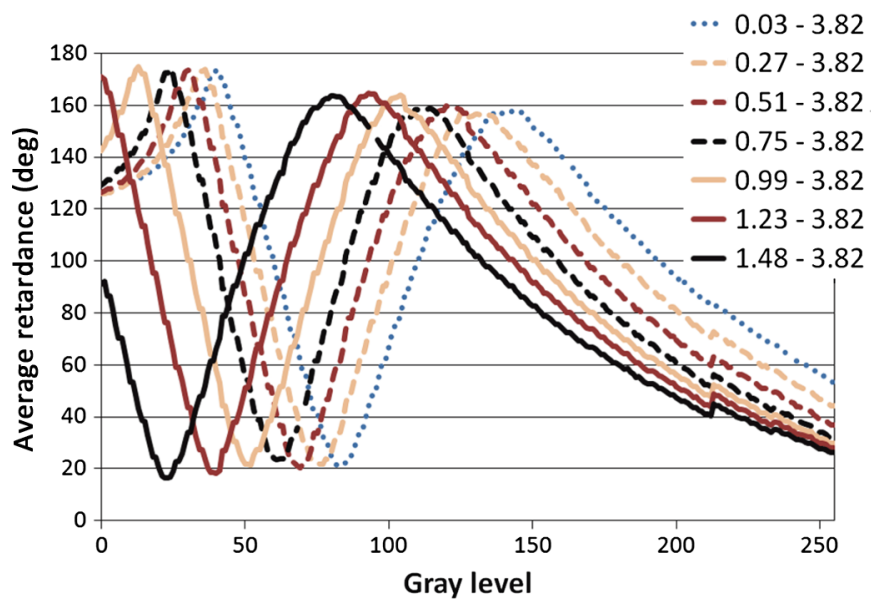

(a)
In Fig. 6(b), as $V_{\text {bright }}$ increases the maxima in the curves shift to lower gray levels, i.e., there is an increase in the accumulated voltage applied associated with each gray level. A combination of the conclusions from Figs. 6(a) and 6(b) provides a guide to tune the parameters $V_{\text {dark }}$ and $V_{\text {bright }}$ to produce specific retardance dynamic range spans as a function of gray level. For example, maximizing the retardance dynamic range means that the accumulated voltage values applied at gray level zero and at high gray levels are, respectively, below LC threshold and close to saturation voltage. To this goal, $V_{\text {dark }}$ must be higher than zero but lower than the threshold and $V_{\text {bright }}$ must be high enough to saturate the LC reorientation: we see in Fig. 6(a) that this is what is obtained with the values $\left(V_{\text {dark }}=0.51 \mathrm{~V}, V_{\text {bright }}=3.82 \mathrm{~V}\right)$ and the maximum retardance span with our LCoS is about $560 \mathrm{deg}$ (unwrapped) at the 633-nm wavelength.

Let us now analyze the retardance difference at the extremals in Figs. 6(a) and 6(b) to learn about the effect of parameters $V_{\text {dark }}$ and $V_{\text {bright }}$ on the flicker magnitude. Comparison between the two maxima exhibited by each of the curves shows that are smaller at the first maximum, occurring at lower gray level values, i.e., lower accumulated voltages. On the other side, if we look at the second maximum, we see that may partly depend on the voltage difference between $V_{\text {bright }}$ and $V_{\text {dark }}$ : a smaller voltage difference seems to produce smaller values, i.e., decrease in the flicker. This can be more clearly analyzed in Figs. 7(a) and 7(b) where the average retardance as a function of gray level is plotted for various combinations of $V_{\text {bright }}$ and $V_{\text {dark }}$ providing different relations of the voltage difference. In Fig. $7(\mathrm{a})$, we see that the curve $(0.81 \mathrm{~V}, 3.16 \mathrm{~V})$ provides a smaller value at the second maximum. In Fig. 7(b), difference $V_{\text {bright }}-V_{\text {dark }}$ is kept constant at the maximum value allowed by the software, which is $3.80 \mathrm{~V}: \Gamma_{\text {diff }}$ remains constant independently of the voltage parameters $V_{\text {bright }}$ and $V_{\text {dark }}$. What is clear from Figs. 6 and 7 is that the retardance difference cannot be diminished from about 20 deg by simply varying $V_{\text {bright }}$ and $V_{\text {dark }}$, so other strategies should be considered, such as the one reported by García-Márquez et al., ${ }^{17}$ consisting of reducing the temperature of the $\mathrm{LCoS}$ to increase the viscosity of the LC thus reducing its time response.

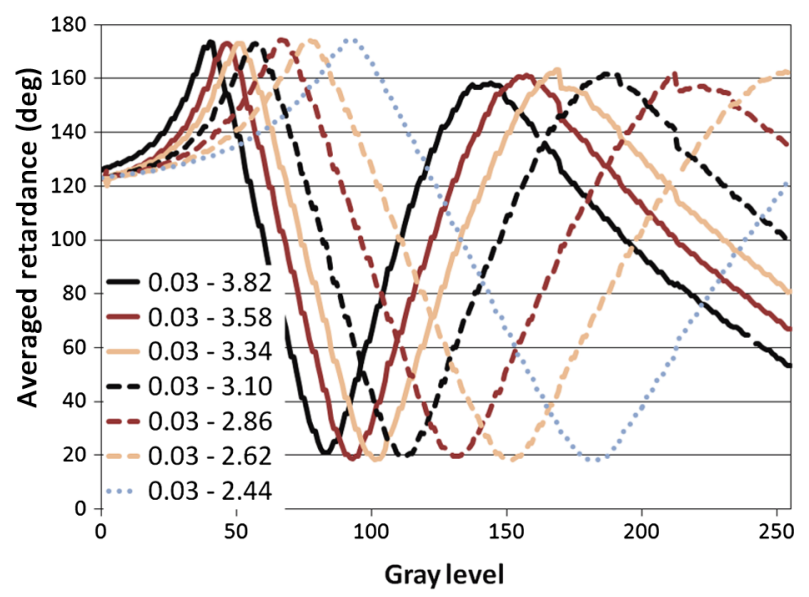

(b)

Fig. 6 Average retardance measured for a series of configurations with $V_{\text {dark }} \neq V_{\text {bright }}:$ (a) changing $V_{\text {dark }}$ $\left(V_{\text {bright }}=3.82 \mathrm{~V}\right)$ and $(\mathrm{b})$ changing $V_{\text {bright }}\left(V_{\text {dark }}=0.03 \mathrm{~V}\right)$. Gamma curve and sequence corresponding to "5-5 default ramp;" $\lambda=633 \mathrm{~nm}$. 


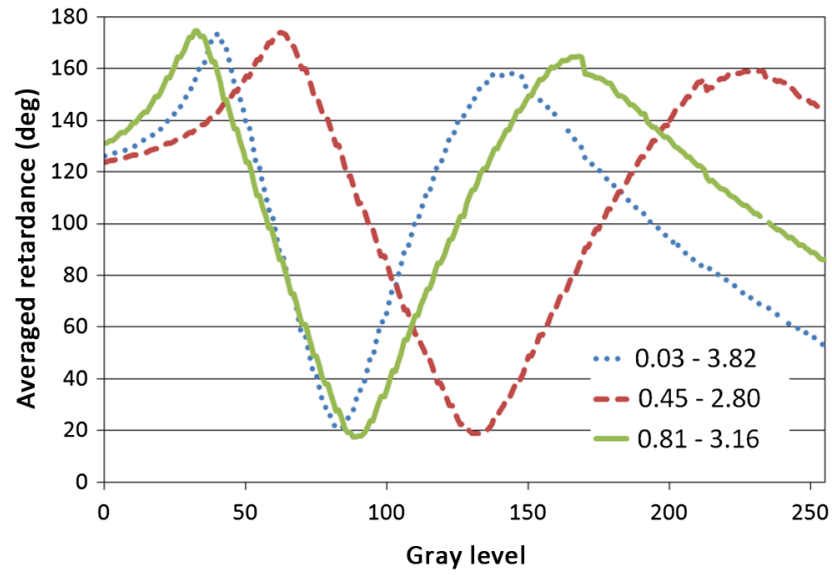

(a)

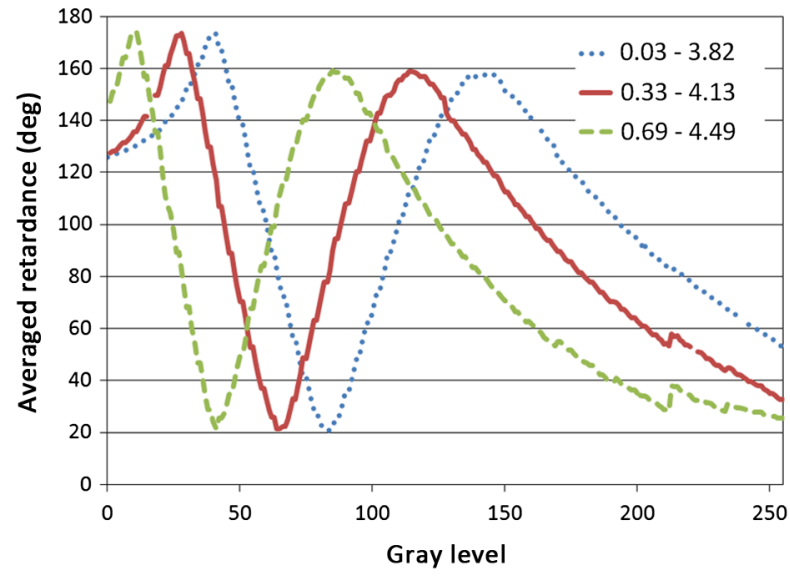

(b)

Fig. 7 Average retardance measured for a series of configurations where the voltage difference between $V_{\text {bright }}$ and $V_{\text {dark }}$ is the magnitude of interest: (a) Different voltage differences and (b) keeping the maximum voltage difference. Gamma curve and sequence corresponding to " $5-5$ default ramp;" $\lambda=633 \mathrm{~nm}$.

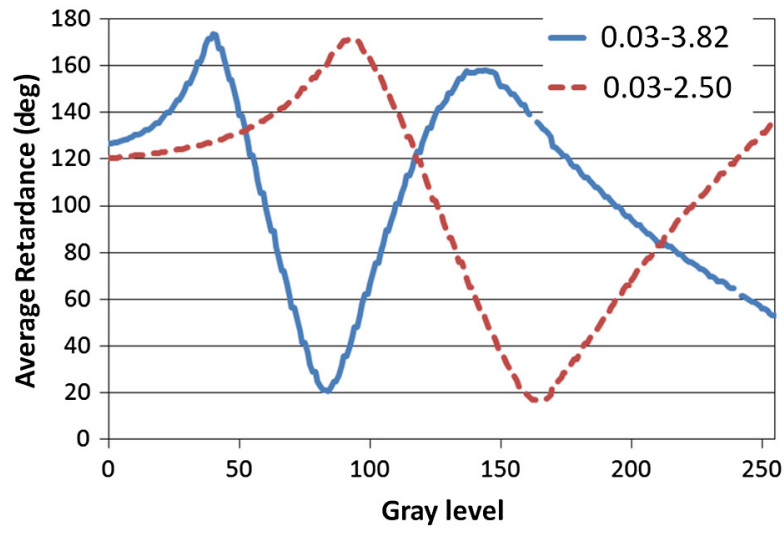

(a)

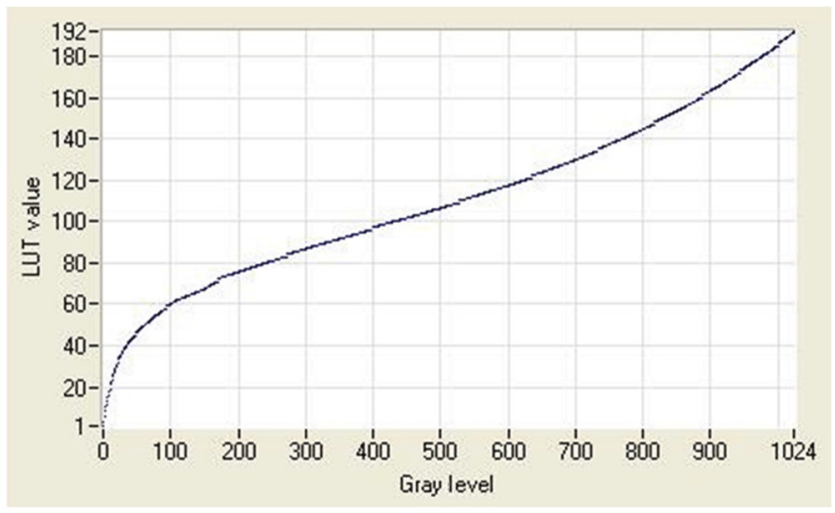

(b)

Fig. 8 (a) Retardance range comparison when $V_{\text {bright }}$ is changed in the "5-5 default ramp" configuration. (b) Linearization look-up table (LUT).

\subsection{Conventional Modulation Regimes and Specialized for Applications}

We can use all the previous analyses to generate the particular modulation configurations needed for each specific application. We will consider two typical cases to exemplify our approach: phase-only modulation and maximum intensity or amplitude-mostly modulation. We want to produce these modulation regimes with a high number of quantization levels, high linearity as a function of gray level, and with minimal flicker influence.

If we want to use the PA-LCoS as a phase-only modulator, linearly polarized light along the director axis must illuminate the device and a 360-deg retardance range as a function of gray level should be produced. This is enabled by configuration "5-5 linear 2pi 633 nm" as shown in Fig. 4(b); however, it provides only 95 out of the 192 possible values which are available for the 5-5 sequence format. To this goal, our starting point is the configuration " $5-5$ default ramp" which covers the maximum retardance range of $560 \mathrm{deg}$. To limit the total range to $360 \mathrm{deg}$, the first step is to accordingly reduce the $V_{\text {bright }}$ voltage. In Fig. 8(a), we show the average retardance for the original and for the reduced voltage range.

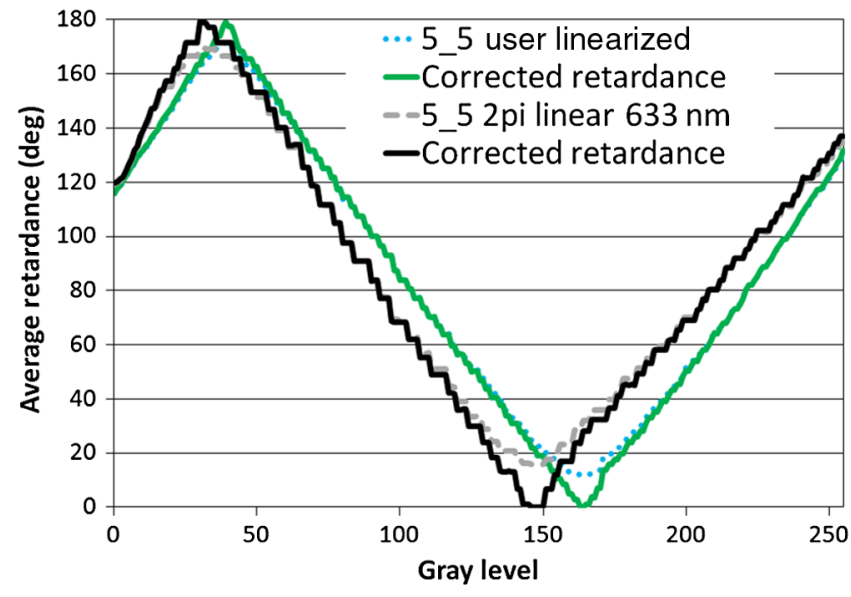

Fig. 9 Configuration for phase-only modulation linearized with the method described, shown against the configuration provided by vendor. 


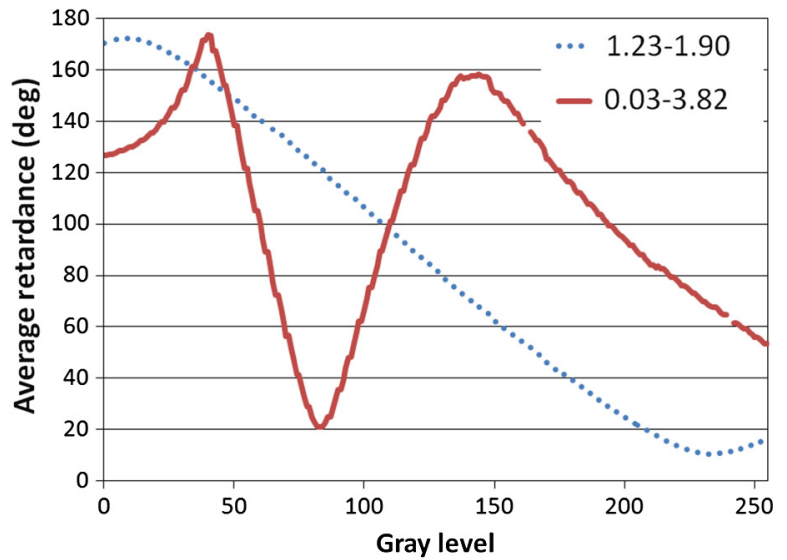

(a)

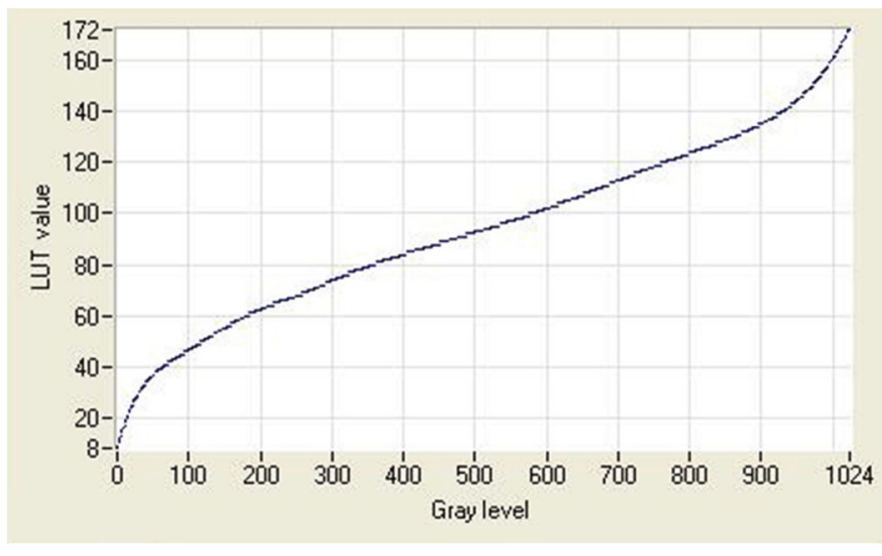

(b)

Fig. 10 (a) Average retardance for the novel intensity modulation configuration compared with the default configuration. (b) Linearization LUT.

Changing $V_{\text {bright }}$ reduces our retardance range to the necessary value, but the curve is highly nonlinear. To linearize the curve first we calculate the corrected retardance by applying the extended calibration method ${ }^{21}$ as was shown in Fig. 4. Then the average retardance and gray level axes are inverted and the corresponding linearization LUT is obtained, shown in Fig. 8(b), where the whole range of 192 quantization levels is maintained.

In Fig. 9, we show the average retardance as a function of gray level for the configuration " $5-5$ linear 2pi $633 \mathrm{~nm}$ " provided by the vendor and the result obtained by applying the configuration we have generated with the method described, which we call "5_5 user linearized." We plot both the uncorrected and the corrected measurements for both configurations. Our novel configuration maintains all the 192 levels available and is highly linear and due to the reduction in the difference between $V_{\text {bright }}$ and $V_{\text {dark }}$, it shows less flicker than the configuration provided by the manufacturer.

Next, we take advantage of the understanding already gained to produce a totally new configuration, able to produce intensity modulation with a high contrast. Now, instead of being linear in average retardance, we need a linear intensity curve as a function of gray level. The starting point is once again the configuration "5-5 default-ramp" which has all the 192 levels available. First of all, we have to reduce

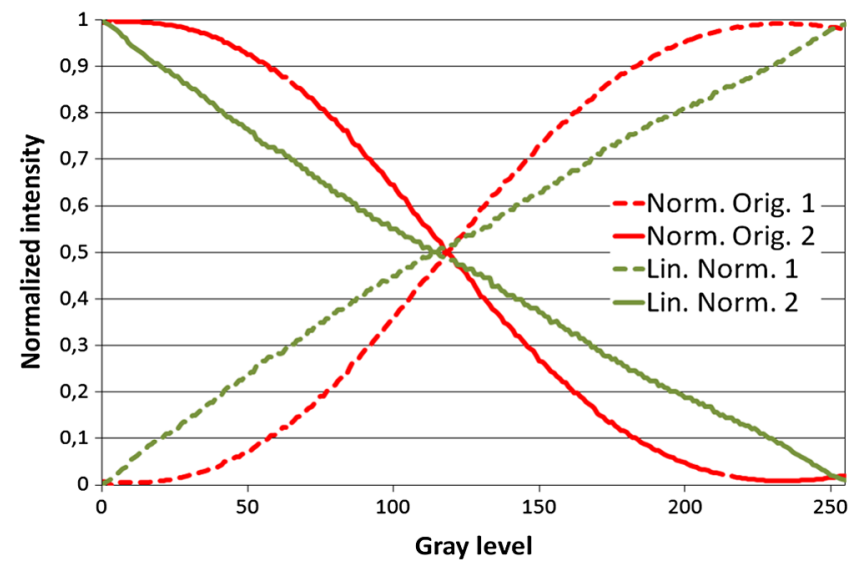

Fig. 11 Intensity versus gray level before and after linearization. the retardance range to $180 \mathrm{deg}$, enough to produce a full swing between zero and maximum intensity modulation for the PA-LCoS inserted between crossed polarizers with their transmission axes at $45 \mathrm{deg}$ with respect to the director axis of the LCoS. This is the actual configuration presented in Sec. 2 for the extended linear polarimeter. We find that applying $V_{\text {dark }}=1.23 \mathrm{~V}$ and $V_{\text {bright }}=1.90 \mathrm{~V}$ reduces the average retardance to a value close to the demanded $180 \mathrm{deg}$ range as we show in Fig. 10(a). Linearization is now obtained by inverting the intensity transmission versus gray level, and the resulting linearization LUT to be applied is shown in Fig. 10(b). The number of quantization levels is 172 , very close to the available 192 . A retardance range slightly larger than $180 \mathrm{deg}$ was previously tolerated and this results in a small cut in the number of quantization levels. Finer adjustment may be possible if necessary.

In Fig. 11, we plot the normalized intensity transmission as a function of gray level obtained with the LCoS between crossed (and parallel) polarizers with their transmission axes at $45 \mathrm{deg}$ with respect to the director axis. We see both the curve before and after the linearization LUT is applied. A very linear curve switching from zero to maximum intensity is obtained showing the feasibility of our proposal.

\section{Conclusions}

In this work, we have applied the extended linear polarimeter method to analyze the influence on the optical signal of the various electrical parameters governing the signal addressed onto digital LCoS devices. The analysis has been performed in terms of the average retardance dynamic range and the magnitude of its flicker. Proper choice of the digital addressing sequence, together with the fine adjustment available via the software options given by the gamma curve and the voltage parameters $V_{\text {dark }}$ and $V_{\text {bright }}$, enable the control of the retardance dynamic range, the linearity in the response of the device, the number of quantization levels, and to some extent the magnitude of the flicker amplitude. Applying the results from this analysis, optimum electrical configurations intended for phase-only and intensity or amplitudemostly modulation regimes, useful in diffractive optics, have been obtained. 


\section{Acknowledgments}

This work was supported by the Ministerio de Trabajo y Competitividad of Spain (projects FIS2011-29803-C02-01 and FIS2011-29803-C02-02), by the Generalitat Valenciana of Spain (projects PROMETEO/2011/021 and ISIC/2012/ 013), and by the Universidad de Alicante (project GRE12-14).

\section{References}

1. J. Turunen and F. Wyrowski, Eds., Diffractive Optics for Industrial and Commercial Applications, Akademie Verlag, Berlin (1997).

2. H. J. Coufal, D. Psaltis, and B. T. Sincerbox, Eds., Holographic Data Storage, Springer-Verlag, Berlin (2000).

3. W. Osten, C. Kohler, and J. Liesener, "Evaluation and application of spatial light modulators for optical metrology," Opt. Pura Apl. 38(3), 71-81 (2005).

4. M. A. F. Roelens et al., "Dispersion trimming in a reconfigurable wavelength selective switch," J. Lightwave Technol. 26(1), 73-78 (2008).

5. M. Salsi et al., "Mode-division multiplexing of $2100 \mathrm{~Gb} / \mathrm{s}$ channels using an LCOS-based spatial modulator," J. Lightwave Technol. 30(4), 618-623 (2012).

6. M. A. Solís-Prosser et al., "Preparing arbitrary pure states of spatial qudits with a single phase-only spatial light modulator," Opt. Lett. 38(22), 4762-4765 (2013).

7. S. T. Wu and D. K. Yang, Reflective Liquid Crystal Displays, John Wiley \& Sons Inc., Chichester (2005).

8. N. Collings et al., "The applications and technology of phase-only liquid crystal on silicon devices," J. Display Technol. 7(3), 112119 (2011).

9. Z. Zhang et al., "High quality assembly of phase-only liquid crystal on silicon (LCOS) devices," J. Display Technol. 7(3), 120-126 (2011).

10. K. Lu and B. E. A. Saleh, "Theory and design of the liquid crystal TV as an optical spatial phase modulator," Opt. Eng. 29(3), 240-246 (1990).

11. A. Márquez et al., "Electrical origin and compensation for two sources of degradation of the spatial frequency response exhibited by liquid crystal displays," Opt. Eng. 46(11), 114001 (2007).

12. L. Lobato et al., "Characterization of the anamorphic and spatial frequency dependent phenomenon in liquid crystal on silicon displays," J. Eur. Opt. Soc. Rapid Pub. 6, 11012S (2011).

13. J. Albero et al., "Second order diffractive optical elements in a spatial light modulator with large phase dynamic range," Opt. Lasers Eng. 51(2), 111-115 (2013)

14. J. E. Wolfe and R. A. Chipman, "Polarimetric characterization of liquid-crystal-on-silicon panels," Appl. Opt. 45(8), 1688-1703 (2006).

15. P. Clemente et al., "Use of polar decomposition of Mueller matrices for optimizing the phase response of a liquid-crystal-on-silicon display," Opt. Express 16(3), 1965-1974 (2008).

16. A. Lizana et al., "Time-resolved Mueller matrix analysis of a liquid crystal on silicon display," Appl. Opt. 47(23), 4267-4274 (2008).

17. J. García-Márquez et al., "Flicker minimization in an LCoS spatial light modulator," Opt. Express 20(8), 8431-8441 (2012).

18. A. Lizana et al., "Influence of the temporal fluctuations phenomena on the ECB LCoS performance," Proc. SPIE 7442, 74420G (2009)

19. A. Hermerschmidt et al., "Wave front generation using a phase-only modulating liquid-crystalbased micro-display with HDTV resolution," Proc. SPIE 6584, 65840E (2007).

20. J. R. Moore et al., "The silicon backplane design for an LCOS polarization-insensitive phase hologram SLM," IEEE Photonic Technol. Lett. 20(1), 60-62 (2008).

21. F. J. Martínez et al., "Extended linear polarimeter to measure retardance and flicker: application to LCOS devices in two working geometries," Opt. Eng. 53(1), 014105 (2014).

22. S. T. Wu, U. Efron, and L. D. Hess, "Birefringence measurements of liquid crystals," Appl. Opt. 23(21), 3911-3915 (1984).

23. A. Márquez et al., "Characterization of the retardance of a wave plate to increase the robustness of amplitude-only and phase-only modulations of a liquid crystal display," J. Mod. Opt. 52(4), 633-650 (2005)

24. E. Lueder, "Liquid crystal on silicon displays," in Liquid Crystal Displays: Addressing Schemes and Electro-Optical Effects," 2nd ed., pp. 353-361, John Wiley \& Sons, Chichester (2010).

25. JVC Newsroom, Electronic Home Expo 2007, Press Release, "DILA $^{\mathrm{TM}}$ Projector Technology: the path to high resolution projection displays," http://pro.jvc.com/pro/pr/2007/ehexpo/dila_wpaper.pdf (1 November 2013)

26. J. Van den Steen, "Design of LCOS microdisplay backplanes for projection applications," pp. 136, Ph.D. Thesis, Gent University, Gent, Belgium (2006).

27. C. Cui-ying et al., "Study of the methods to realize gray scale for $\mathrm{LCoS}$ based on sub-frame modulation," in Proc. of the Symposium on Photonics and Optoelectronics (SOPO), IEEE, New York (2011).

28. J.-S. Kang and O.-K. Kwon, "Digital driving method for low frame frequency and 256 gray scales in liquid crystal on silicon panels," J. Display Technol. 8(12), 723-729 (2012).
29. G. Lazarev et al., "LCOS spatial light modulators: trends and applications," in Optical Imaging and Metrology: Advanced Technologies, W. Osten and N. Reingand, Eds., pp. 1-29, John Wiley \& Sons, Weinheim (2012).

30. C. Ramirez et al., "Point diffraction interferometer with a liquid crystal monopixel," Opt. Express 21(7), 8116-8124 (2013).

31. A. Márquez et al., "Study of the modulation capabilities of parallel aligned liquid crystal on silicon displays," Proc. SPIE 8855, 885504 (2013).

32. C. Ramirez et al., "Polarimetric method for liquid crystal displays characterization in presence of phase fluctuations," Opt. Express 21(3), 3182-3192 (2013)

33. F. J. Martínez et al., "Retardance and flicker modeling and characterization of electrooptic linear retarders by averaged Stokes polarimetry," Opt. Lett. 39(4), 1011-1014 (2014).

34. Jasper Display Corporation News, "FPD 2012 Jasper Announced 1080p LCOS for phase modulation," http://www.jasperdisplay.com/ news/detail/308/ (1 November 2013).

35. A. Lizana et al., "The minimum Euclidean distance principle applied to improve the modulation diffraction efficiency in digitally controlled spatial light modulators," Opt. Express 18(10), 1058110593 (2010).

Francisco Javier Martínez received his BS degree in electronic engineering from University of Valencia in 1996 and his BS degree in physics in 1999. Currently, he is a research assistant with the Applied Physics to Science and Technology Institute from University of Alicante, and assistant professor of electronics at Miguel Hernandez University. His research interests include diffractive optics and holography.

Andrés Márquez received his $\mathrm{MSc}$ and $\mathrm{PhD}$ degrees in physics from Universidad Autónoma de Barcelona in 1997 and 2001, respectively. In 2000, he joined the Universidad de Alicante, where he is an associate professor of applied physics. His research interests include holographic recording materials, liquid crystal spatial light modulators, optical image processing, and diffractive optics.

Sergi Gallego obtained his Msc degree in physics at University of Valencia in 2001 and his PhD degree at the University of Alicante, Spain, in 2005, where he works as a lecturer. His research interests include holographic recording materials, diffraction, liquid crystal displays applied to holography, diffractive elements, and photopolymers. He has authored or coauthored 1 patent and more than 60 publications in renowned international journals.

Manuel Ortuño received his MSc degree in organic chemistry from the University of Murcia, Spain, in 1993. He received his MSc degree in chemical engineering in 1999 and a PhD degree in physics from the University of Alicante, Spain, in 2005. In 2004, he joined the Universidad de Alicante, where he is an associate professor. His research interests include light sensitive materials, photopolymers, holographic recording materials, liquid crystals, dyes and green materials.

Jorge Francés received a $\mathrm{PhD}$ degree at the University of Alicante in 2011. He received his MSEE in 2009 and his BSEE in 2006, both from the Technical University of Valencia, Valencia, Spain. He has been working as an assistant lecturer with the University of Alicante since 2008. His main research interests include physical optics, sound and vibration, and numerical simulation.

Augusto Beléndez received his MSc degree and $\mathrm{PhD}$ degree in physics from the University of Valencia, Spain, in 1986 and 1990, respectively. In 1996 he became full professor of applied physics at the University of Alicante. He is mainly interested in holography, holographic recording materials, holographic optical elements, optical processing, nonlinear oscillations and teaching of physics and engineering. He has published more than 250 technical papers and presented more than 210 papers at scientific conferences.

Inmaculada Pascual received her MSc degree in physics from University of Granada in 1985 and her PhD degree from University of Valencia in 1990. She is a full professor of optics at the University of Alicante. She has carried out research in holography, mainly on holographic recording material, holographic optical elements, and optical data storage. She has published more than 125 papers and presented more than 170 papers at scientific conferences. 